\title{
Adaptive Optics observations of the Galactic center young stars
}

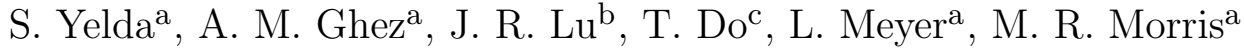 \\ aUCLA Department of Physics and Astronomy, Los Angeles, CA 90095; \\ ${ }^{b}$ Institute for Astronomy, University of Hawaii, Honolulu, HI, 96822; \\ ${ }^{c}$ UCI Department of Physics and Astronomy, Irvine, CA 92697
}

\begin{abstract}
Adaptive Optics observations have dramatically improved the quality and versatility of high angular resolution measurements of the center of our Galaxy. In this paper, we quantify the quality of our Adaptive Optics observations and report on the astrometric precision for the young stellar population that appears to reside in a stellar disk structure in the central parsec. We show that with our improved astrometry and a 16 year baseline, including 10 years of speckle and 6 years of laser guide star AO imaging, we reliably detect accelerations in the plane of the sky as small as $70 \mu \mathrm{as} \mathrm{yr}^{-2}\left(\sim 2.5 \mathrm{~km} \mathrm{~s}^{-1} \mathrm{yr}^{-1}\right)$ and out to a projected radius from the supermassive black hole of $1^{\prime \prime} .5(\sim 0.06 \mathrm{pc})$. With an increase in sensitivity to accelerations by a factor of $\sim 6$ over our previous efforts, we are able to directly probe the kinematic structure of the young stellar disk, which appears to have an inner radius of 0.8 . We find that candidate disk members are on eccentric orbits, with a mean eccentricity of $\langle e\rangle=0.30 \pm 0.07$. Such eccentricities cannot be explained by the relaxation of a circular disk with a normal initial mass function, which suggests the existence of a top-heavy IMF or formation in an initially eccentric disk.
\end{abstract}

Keywords: adaptive optics, astrometry

\section{INTRODUCTION}

The proximity of the Galactic center (GC) makes the region uniquely suited for the study of a supermassive black hole and its effects on the surrounding nuclear stellar cluster. Advancements in technology, in particular adaptive optics (AO) systems on large (8-10 m), ground-based telescopes, have afforded us a view of this dense region at such high angular resolution that individual stellar orbits of short-period stars can be precisely measured. The orbit of the central arcsecond star, S0-2, for example, has provided the best evidence to date that supermassive black holes (SMBH) exist at the centers of normal galaxies, and that the black hole at the Galactic center, Sgr $\mathrm{A}^{*}$, has a mass of $M_{B H}=4.1 \times 10^{6} \mathrm{M}_{\odot}$ and distance of $R_{o}=8.0 \mathrm{kpc}$, respectively ${ }^{1 / 2}$

With high precision astrometric and spectroscopic measurements, much can also be understood about the nuclear star cluster in the vicinity of the SMBH. While the GC is populated predominantly by old, late-type stars ( 1 Gyr), spectroscopic observations of the region have unveiled $\sim 200$ massive, young ( 6 Myr) stars in the central parsec ${ }^{3-7}$ whose origins are a puzzle due to the strong tidal forces from the black hole. Given the youth of this population and the relaxation timescale in the GC $(\sim 1 \mathrm{Gyr}), \frac{8}{8}$ the stars' dynamical properties contain a signature of their formation history. A particularly prominent feature that has been observed is a stellar disk containing a significant fraction (possibly up to 50\%) of the young stars orbiting the black hole in a clockwise sense. ${ }^{[6]-13]}$ The orientation of the disk orbital plane can be described by its inclination and angle to the ascending node, and is roughly $i \sim 130^{\circ}$ and $\Omega \sim 100^{\circ}$, with a $1 \sigma$ dispersion of $\sim 10^{\circ}$.6[12]13] The disk's surface density profile has been observed to fall off like $R^{-2}$, which has been the main argument for in situ formation and against cluster infall. $6[12,13$

While there is consensus in the literature regarding the existence of the clockwise disk and its surface density profile, many of its properties have yet to be constrained, in part because interpretations of kinematic studies rely on the ability to assign disk membership. The difficulty in determining which stars belong to the disk

Further author information: (Send correspondence to Sylvana Yelda)

E-mail: syelda@astro.ucla.edu 
arises from the lack of knowledge about a star's line-of-sight distance relative to Sgr A*. This can be overcome, however, with detections of accelerations in the plane of the sky 12$]$ While some stars in the central arcsecond $\left(r<00^{\prime \prime} 5\right)$ have been seen to orbit Sgr A* completely, ${ }^{[12}$ the detection of accelerations becomes more difficult at larger radii where the clockwise disk stars are located $(r>0$ "' 8$)$ and requires both high precision astrometry and patience. In this proceeding, we report on the quality of our $\mathrm{AO}$ observations (\$2), our astrometric precision, including detections of accelerations in the plane of the sky for the young stars (\$3), and finally the eccentricity distribution of the accelerating disk stars (\$4).

\section{HIGH ANGULAR RESOLUTION IMAGING}

The Galactic center region was imaged at high angular resolution with the Keck telescopes from 1995-2011. In the first decade, the images were made with the near-infrared speckle imaging camera, which provided diffraction limited images $\left(\theta=00^{\prime \prime} 05\right)$ with Strehl ratios of $\sim 0.05$. In each final image constructed from the speckle data $\left(<N_{\text {exp }}>\sim 1600, t_{\text {exp }} \sim 0.14 \mathrm{~s}\right.$, coadd $\left.=1\right)$, we detect $\sim 160$ stars on average, down to $K \sim 16$ in a $55^{\prime \prime} 2 \times 5^{\prime \prime} 2$ field of view.

Starting in 2004, images were obtained with the Keck II laser guide star adaptive optics system in conjunction with the facility near infrared camera, NIRC2 $\left(<N_{\text {exp }}>\sim 100, t_{\text {exp }} \sim 2.8 \mathrm{~s}\right.$, coadd $\left.=10\right)$. The tip/tilt star is located at a distance of $r=19^{\prime \prime}$ from the GC. Despite this relatively large distance, as compared with speckle imaging these data provide nearly an order of magnitude improvement in Strehl ratio $(\sim 0.28)$, a factor of 4 increase in areal coverage (field of view of $10^{\prime \prime} \times 10^{\prime \prime}$ ) and a considerable increase in the average number of detected stars $(\sim 2000)$ down to $K \sim 20$. Details of these observations can be found in Ghez et al., 1,10 et al., 12 and Yelda et al. (in preparation). With these data, we measure the proper motions and accelerations in the plane of the sky for a sample of $\sim 70$ young stars, which have known line of sight velocities $\left(v_{z}\right)^{6 / 13 \sqrt[14]{14}}$ and which are located at projected radii $r>00^{\prime \prime} 8-7^{\prime \prime}$.

\section{HIGH PRECISION ASTROMETRY}

There are several sources of statistical uncertainty in the positional measurements that must be accounted for. While speckle images have typical centroiding uncertainties of $\sigma_{\text {pos }} \sim 0.85$ mas, the high angular resolution AO images allow for nearly an order of magnitude improvement for the brightest stars with $\sigma_{\text {pos }} \sim 0.1$ mas (red squares in Figure 1). This improvement is a result of the higher signal to noise ratio in the AO images. A second source of uncertainty comes from the transformation process that aligns the stars' positions across all epochs $\left(\sigma_{a l n}\right)$. These alignment errors are a function of time from the reference epoch and of the number of reference stars used in the transformation to the so-called "maser-frame" in which Sgr A* is at rest (see Yelda et al! $\frac{15}{15}$ for details of this process). As seen in Figure 1 , $\sigma_{a l n}$ is minimized near the reference epoch, 2006 June, and is larger for the speckle epochs $\left(\sigma_{a l n}>0.5\right.$ mas), which have on average $\sim 6 \times$ fewer reference stars than are available in $\mathrm{AO}$ epochs. Finally, there is also a source of statistical uncertainty in the positional measurements that is not captured in the other terms that arises from inaccuracies in the estimates of the PSF wings of neighboring sources. This additive error term $\left(\sigma_{a d d}\right)$ is estimated as the amount of error required to minimize the residuals between the observed and expected velocity $\chi^{2}$ distribution for a given number of degrees of freedom (see Clarkson et al ${ }^{16}$ and Yelda et al. (in preparation) for details). The size of this error term is negligible for the speckle data ( $\sigma_{\text {add }}=0.18$ mas), but becomes important for the $\mathrm{AO}$ images $\left(\sigma_{\text {add }}=0.10\right.$ mas).

Positions as a function of time are fit for proper motions and accelerations. Whether a star exhibits significant accelerated motion in the plane of the sky depends on several factors, including its distance from the supermassive black hole, the time baseline over which it is detected, and the precision with which its positions are measured. In order to determine whether a star's motion is best described by a velocity or acceleration model, we first compare the goodness of fit of the two models using the F-test, in which we estimate the quantity $F=\chi_{p m}^{2} / \chi_{a c c}^{2}$, which follows an F-distribution under the null hypothesis ${ }^{17}$ Here $\chi_{p m}^{2}$ and $\chi_{a c c}^{2}$ are the reduced $\chi^{2}$ values for the proper motion and acceleration fits, respectively. We require that $\mathrm{F}$ is greater than the critical $\mathrm{F}$ value at the $4 \sigma$ significance level and that a star is detected in more than 30 out of the 45 possible epochs, which removes the sources that show unphysical accelerations arising from effects such as source confusion or edge effects in speckle images. The resulting six stars have reliable radial acceleration measurements at $>5$ sigma. For all other sources, the proper motion fit is used. 


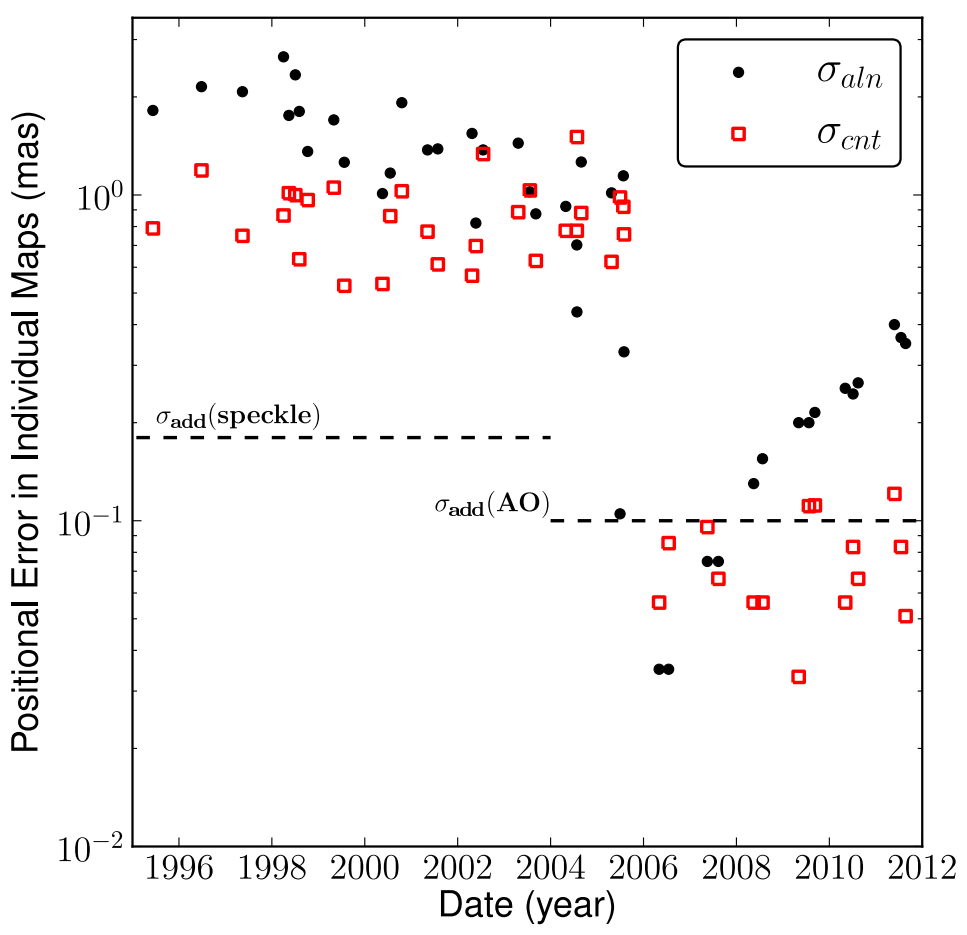

Figure 1. Alignment (filled black points) and centroiding (unfilled red squares) uncertainties as a function of epoch. The median uncertainty of the young stars is reported for each epoch. Alignment errors are minimized near the reference epoch, 2006 June, and increase with time away from this epoch. All epochs with $\sigma_{a l n}>0.5$ mas are from speckle imaging, where the higher uncertainties are a result of very few reference stars as compared to AO data. The additive errors for speckle and $\mathrm{AO}$ are shown as dashed lines.

The position, proper motion, and acceleration errors from the fitting procedure as a function of $\mathrm{K}$ magnitude $(\lambda=2.2 \mu \mathrm{m})$ are shown in Figure 2 for our sample of young stars. The median errors in positions and proper motions are 0.05 mas and 0.03 mas $\mathrm{yr}^{-1}$, respectively. Stars detected in relatively more epochs show smaller errors in positions and velocities, as seen in the top panels of Figure 2. Acceleration errors are plotted for the six stars that have reliable acceleration measurements, as well as 11 stars with $3 \sigma$ upper limits below their theoretical

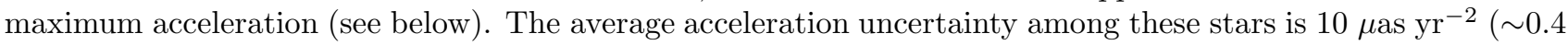
$\mathrm{km} \mathrm{s}^{-1} \mathrm{yr}^{-1}$ ), which is a factor of six improvement over our earlier efforts in $\mathrm{Lu}$ et al ${ }^{12}$

Figure 3 shows the radial acceleration measurements as a function of projected radius for stars in the central $10^{\prime \prime}$ field for which we have acceleration information. The six significant accelerations are shown with $1 \sigma$ error bars. This increases the number of acceleration measurements beyond $1^{\prime \prime}$ over our previous work in Lu et al ${ }^{12}$ by a factor of six, or equivalently, an additional five stars, three of which are reported by Gillessen et al ${ }^{2}$ The most distant star from the SMBH for which an acceleration measurement is made is IRS 16SW, located at $R=$ $1^{\prime \prime} 5$ ( $\sim 0.06 \mathrm{pc}$ ), which is well outside the inner edge of the stellar disk. An additional 11 stars have $3 \sigma$ upper limits below the theoretical maximum acceleration given by each star's projected radius, thereby requiring a non-zero line-of-sight distance.

\section{ORBITS OF THE GALACTIC CENTER YOUNG STARS}

With six kinematic variables measured $\left(x_{0}, y_{0}, v_{x}, v_{y}, v_{z}, a_{R}\right)$, the orbital elements can be estimated if the black hole properties are known. We use updated SMBH properties, which are described in detail in Yelda et al. (in preparation). We estimate uncertainties in the orbital period $(P)$, eccentricity $(e)$, inclination $(i)$, angle to the ascending node $(\Omega)$, longitude of periapse $(\omega)$, and time of periapase passage $\left(T_{0}\right)$ by carrying out a 

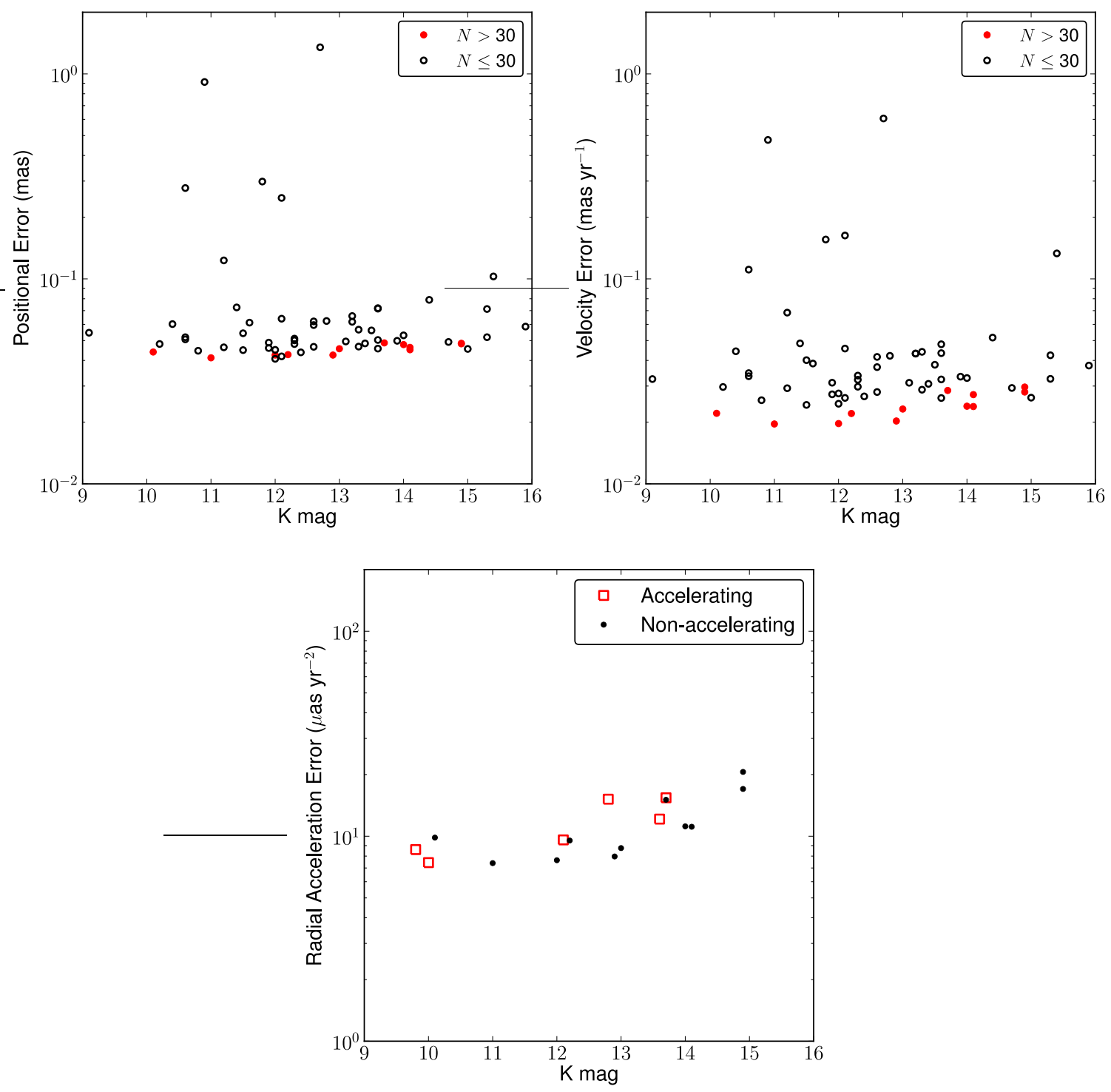

Figure 2. Position (top left), proper motion (top right), and radial acceleration errors (bottom) as a function of K magnitude for our sample of young stars beyond a projected radius of 0. . 8. Errors are estimated from either the proper motion or acceleration fit to each star's individual positions over time. Stars with $N>30$ epochs (filled red points) have smaller errors in position and proper motion than stars in fewer epochs (open circles). The radial acceleration errors are shown for stars in $N>30$ epochs, where we separately plot those six stars passing the F test for accelerations (open red squares) and those stars for which no detectable accelerations are found (filled black points), requiring a non-zero line-of-sight distance.

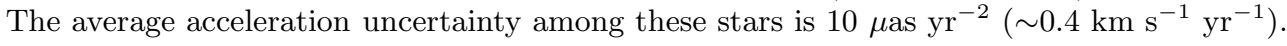

Monte Carlo simulation in which we create $10^{5}$ artificial data sets. In each data set, we sample the six kinematic measurements, as well as the gravitational potential parameters (the black hole's mass, distance, position, and an extended mass component). For stars without $a_{R}$, we assume a uniform acceleration prior, with the minimum acceleration set by assuming the stars are bound and the maximum acceleration set by the stars' projected radii. Radial velocities, $v_{z}$, are measured with the Keck AO-assisted integral field spectrograph, OSIRIS, 14 or taken from the literature if needed.6|[13 The kinematic variables are transformed to six orbital elements using the analytic orbit equations presented in Lu et al ${ }^{12}$ 


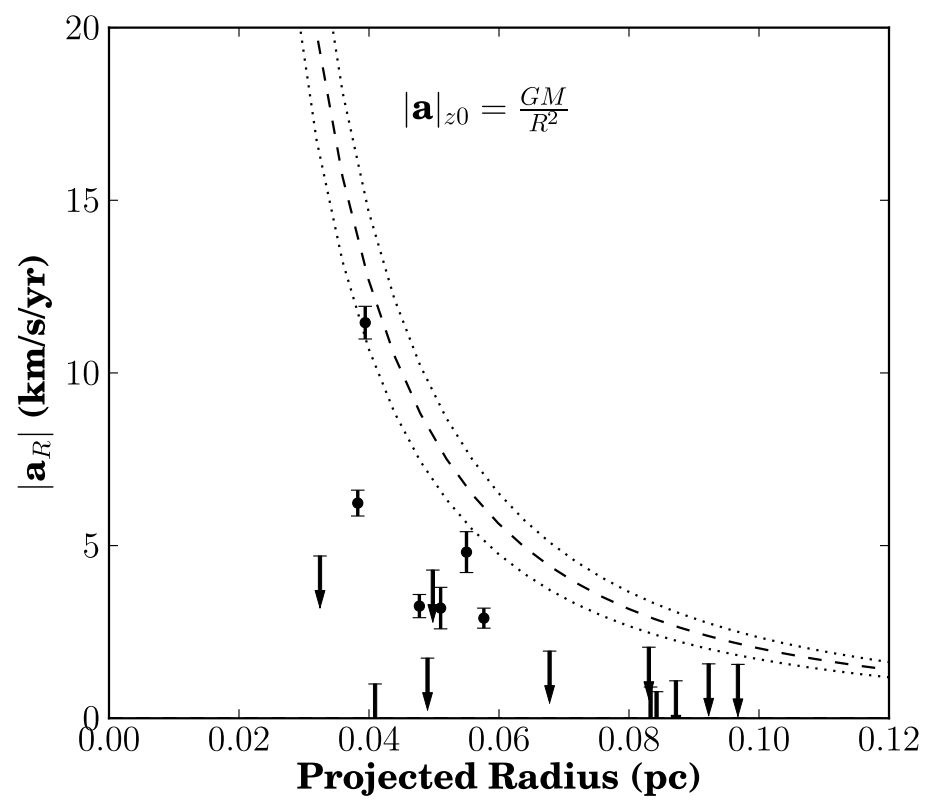

Figure 3. Accelerations along the radial coordinate as a function of projected radius. The theoretical maximum acceleration $\left(|a|_{z 0}\right)$ for the black hole mass of $4.6 \times 10^{6} \mathrm{M}_{\odot}$ (see Yelda et al., in preparation) is shown as the dashed curve, with the $1 \sigma$

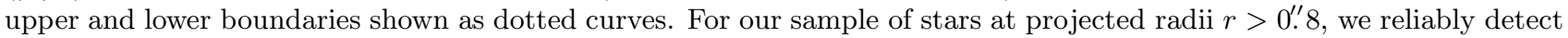
six significant accelerations out to $\mathrm{R}=1^{\prime \prime} .5(0.06 \mathrm{pc})$, shown with $1 \sigma$ error bars. These accelerations yield line-of-sight distances to these sources, allowing for precise determination of the stars' orbits. Stars with $3 \sigma$ acceleration upper limits below the theoretical maximum acceleration $(+1 \sigma)$ are shown as downward pointing arrows and have strong constraints on their line-of-sight distances $(|z|>0)$.

For the six accelerating stars, their line-of-sight distances are determined to within $\sim 0.01 \mathrm{pc}(1 \sigma)$ and therefore result in precisely determined orbital parameter estimates. Each of these sources has an inclination, $i$, and angle to the ascending node, $\Omega$, that is consistent with the orientation of the clockwise disk ${ }^{6 / 12}[13$ making all six stars candidate disk members (Yelda et al., in preparation). Figure 4 shows the combined eccentricity distribution for these sources, where only disk solutions (i.e., those solutions that are within $15^{\circ}$ from the orientation of the disk) are included. The orbits for these stars have a mean eccentricity of $\langle e\rangle=0.30 \pm 0.07$.

\section{CONCLUSIONS}

Adaptive optics observations have provided a substantial leap forward in the quality of Galactic center measurements. As compared to speckle imaging observations, nearly an order of magnitude improvement is seen in Strehl ratio, number of stars detected, and astrometric precision. Such high astrometric precision of stars in the GC has led to detections of accelerations in the plane of the sky, which thereby give a measurement of line-of-sight distances from the black hole. We present the accelerations and eccentricity distribution for six young stars, which are all candidate members of the clockwise stellar disk in the central parsec. The mean eccentricity of the disk stars is $\langle e\rangle=0.30 \pm 0.07$. Given the age of these stars, self-relaxation in an initially circular disk with a normal IMF cannot excite the orbital eccentricities to such high values. Alexander et al ${ }^{18}$ showed that such eccentricities can be reached only if the IMF were extremely top-heavy. Although some authors have claimed evidence for a top-heavy IMF, ${ }^{\mid 19}$ the matter is still under debate (Lu et al., in preparation). Alternatively, the stars could have formed in an originally eccentric disk.

Astrometric measurements of the Galactic center are currently limited by knowledge of the point spread function, which has been assumed to be constant across the image. However, both instrumental and anisoplanatic effects lead to a spatially- and temporally-variable PSF. Our group is currently developing a methodology to accurately model both the static, instrumental PSF (see Fitzgerald et al., this volume) and the time-variable PSF 


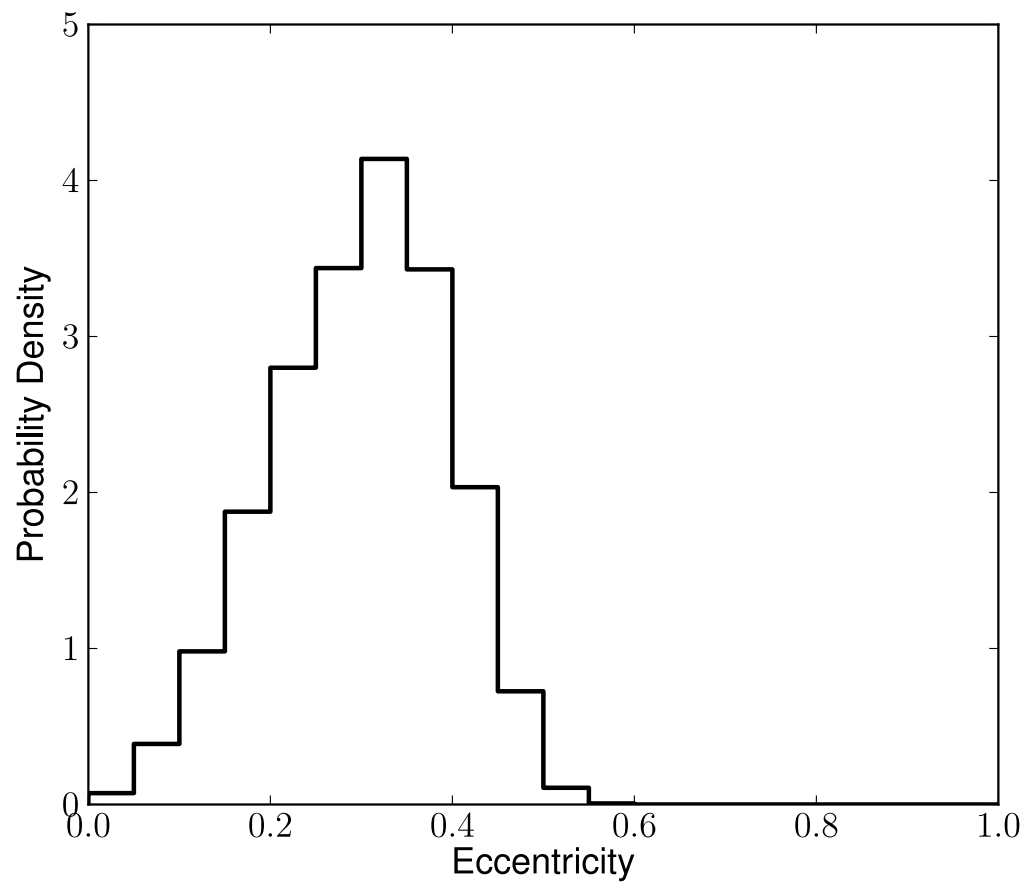

Figure 4. Eccentricity distribution from $10^{5}$ Monte Carlo trials for the six accelerating stars. Solutions that are within $15^{\circ}$ of the clockwise disk orientation are included. All six stars have orbital planes that are consistent with the clockwise disk. These candidate disk stars have eccentric orbits, with an average eccentricity of $0.30 \pm 0.07$.

using atmospheric profiler data taken simultaneously with our science observations. Accurate PSF modeling will allow for an improved optical distortion model and a more stable astrometric reference frame, which is critical for detecting the effects of general relativity and extended mass on the orbits of short-period stars at the GC!

\section{REFERENCES}

[1] Ghez, A. M., Salim, S., Weinberg, N. N., Lu, J. R., Do, T., Dunn, J. K., Matthews, K., Morris, M. R., Yelda, S., Becklin, E. E., Kremenek, T., Milosavljevic, M., and Naiman, J., "Measuring Distance and Properties of the Milky Way's Central Supermassive Black Hole with Stellar Orbits," ApJ 689, 1044-1062 (Dec. 2008).

[2] Gillessen, S., Eisenhauer, F., Trippe, S., Alexander, T., Genzel, R., Martins, F., and Ott, T., "Monitoring Stellar Orbits Around the Massive Black Hole in the Galactic Center," ApJ 692, 1075-1109 (Feb. 2009).

[3] Allen, D. A., Hyland, A. R., and Hillier, D. J., "The source of luminosity at the Galactic Centre," $M N$ RAS 244, 706-713 (June 1990).

[4] Najarro, F., Krabbe, A., Genzel, R., Lutz, D., Kudritzki, R. P., and Hillier, D. J., "Quantitative spectroscopy of the HeI cluster in the Galactic center.," AAP 325, 700-708 (Sept. 1997).

[5] Ghez, A. M., Becklin, E., Duchjne, G., Hornstein, S., Morris, M., Salim, S., and Tanner, A., "Full Three Dimensional Orbits For Multiple Stars on Close Approaches to the Central Supermassive Black Hole," Astronomische Nachrichten Supplement 324, 527-533 (Sept. 2003).

[6] Paumard, T., Genzel, R., Martins, F., Nayakshin, S., Beloborodov, A. M., Levin, Y., Trippe, S., Eisenhauer, F., Ott, T., Gillessen, S., Abuter, R., Cuadra, J., Alexander, T., and Sternberg, A., "The Two Young Star Disks in the Central Parsec of the Galaxy: Properties, Dynamics, and Formation," ApJ 643, 1011-1035 (June 2006). 
[7] Bartko, H., Martins, F., Trippe, S., Fritz, T. K., Genzel, R., Ott, T., Eisenhauer, F., Gillessen, S., Paumard, T., Alexander, T., Dodds-Eden, K., Gerhard, O., Levin, Y., Mascetti, L., Nayakshin, S., Perets, H. B., Perrin, G., Pfuhl, O., Reid, M. J., Rouan, D., Zilka, M., and Sternberg, A., "An Extremely Top-Heavy Initial Mass Function in the Galactic Center Stellar Disks," ApJ 708, 834-840 (Jan. 2010).

[8] Hopman, C. and Alexander, T., "Resonant Relaxation near a Massive Black Hole: The Stellar Distribution and Gravitational Wave Sources," ApJ 645, 1152-1163 (July 2006).

[9] Genzel, R., Pichon, C., Eckart, A., Gerhard, O. E., and Ott, T., "Stellar dynamics in the Galactic Centre: proper motions and anisotropy," MNRAS 317, 348-374 (Sept. 2000).

[10] Levin, Y. and Beloborodov, A. M., "Stellar Disk in the Galactic Center: A Remnant of a Dense Accretion Disk?," ApJL 590, L33-L36 (June 2003).

[11] Genzel, R., Schödel, R., Ott, T., Eisenhauer, F., Hofmann, R., Lehnert, M., Eckart, A., Alexander, T., Sternberg, A., Lenzen, R., Clénet, Y., Lacombe, F., Rouan, D., Renzini, A., and Tacconi-Garman, L. E., "The Stellar Cusp around the Supermassive Black Hole in the Galactic Center," ApJ 594, 812-832 (Sept. 2003).

[12] Lu, J. R., Ghez, A. M., Hornstein, S. D., Morris, M. R., Becklin, E. E., and Matthews, K., "A Disk of Young Stars at the Galactic Center as Determined by Individual Stellar Orbits," ApJ 690, 1463-1487 (Jan. 2009).

[13] Bartko, H., Martins, F., Fritz, T. K., Genzel, R., Levin, Y., Perets, H. B., Paumard, T., Nayakshin, S., Gerhard, O., Alexander, T., Dodds-Eden, K., Eisenhauer, F., Gillessen, S., Mascetti, L., Ott, T., Perrin, G., Pfuhl, O., Reid, M. J., Rouan, D., Sternberg, A., and Trippe, S., "Evidence for Warped Disks of Young Stars in the Galactic Center," ApJ 697, 1741-1763 (June 2009).

[14] Do, T., Ghez, A. M., Morris, M. R., Lu, J. R., Matthews, K., Yelda, S., and Larkin, J., "High Angular Resolution Integral-Field Spectroscopy of the Galaxy's Nuclear Cluster: A Missing Stellar Cusp?," ApJ 703, 1323-1337 (Oct. 2009).

[15] Yelda, S., Lu, J. R., Ghez, A. M., Clarkson, W., Anderson, J., Do, T., and Matthews, K., "Improving Galactic Center Astrometry by Reducing the Effects of Geometric Distortion," ApJ 725, 331-352 (Dec. 2010).

[16] Clarkson, W., Ghez, A., Morris, M., Lu, J., Stolte, A., McCrady, N., Do, T., and Yelda, S., "Proper motions of the Arches cluster with Keck-LGS Adaptive Optics: the first kinematic mass measurement of the Arches," ArXiv e-prints (Dec. 2011).

[17] Hays, W. L., [Statistics], Harcourt Brace College Publishers (1994).

[18] Alexander, R. D., Begelman, M. C., and Armitage, P. J., "Constraints on the Stellar Mass Function from Stellar Dynamics at the Galactic Center," ApJ 654, 907-914 (Jan. 2007).

[19] Nayakshin, S. and Sunyaev, R., "The 'missing' young stellar objects in the central parsec of the Galaxy: evidence for star formation in a massive accretion disc and a top-heavy initial mass function," MNRAS 364, L23-L27 (Nov. 2005). 